\title{
Б.И. Пелешенко
}

\section{Днепропетровский государстьенный аграрный университет}

\section{Об условиях ограниченности операторов свертки в симметрических пространствах}

Исследуется взаимосвязь между достаточными условиями типа Хермандера и Харди - Литтлвуда - Соболева ограниченности иятегральных операторов свертки в симметричньх пространствах.

Пусть $\Phi$ - множество таких вогнутых возрастающих на полуоси $[0, \infty)$ функций $\varphi(t)$, что $\varphi(0)=0$ и $\varphi(t) \rightarrow \infty$ при $t \rightarrow \infty ; \alpha_{\varphi}$ и $\beta_{\varphi}-$ нижний и верхний показатели растяжения ' Һункции $\varphi(t) \in \Phi$. Через $g^{*}(t)$ обозначается невозрастающая перестаноха модуля измеримой на $\mathrm{R}^{\mathrm{n}}$ функции $\mathrm{g}(\mathrm{x}), \mathrm{g}{ }^{* *}(\mathrm{t})=\frac{1}{\mathrm{t}} \int_{0}^{\mathrm{t}} \mathrm{g}^{*}(\tau) \mathrm{d} \tau$. Пусть $\mathrm{L}_{\mathrm{p}}\left(\mathrm{S}^{\mathrm{n}}\right), 1 \leq \mathrm{p}<\infty,-$ лебт: >во пространство интегрируемых в $\mathrm{p}$-й степени на $\mathrm{R}^{\mathrm{n}}$ вецественных функций, $M_{\psi}\left(R^{n}\right)$ и $\Lambda_{\varphi}\left(R^{n}\right)$ - пространства Марцинкевиэа и Лоренца соответственно с фундаментальными функциями $\mathrm{t} / \psi(\mathrm{t})$ и $\varphi(\mathrm{t})[1]$.

Наконец $\chi_{B_{3}}(x)$ обозначает характеристическую функцию куба $\mathrm{B}_{\mathrm{s}} \in \mathrm{R}^{\mathrm{n}}$ с центром в начале координат и длинсй ребра $\mathrm{s}>0$.

Теорема 1. Пусть показатели растяжения вогкутьх функций $\varphi_{0}(t)$, $\varphi(t)$ из $\Phi$ удовлетворяют условию $0<\alpha_{\varphi}, \beta_{\varphi_{0}}<1$, а фуункция $\varphi(t) / \varphi_{0}(t)$ монотонно возрастает. Если локально интегрируемая функция $\mathrm{K}(\mathrm{x})$ такова, что для любого $\mathrm{r}>0$ и всех $|\mathrm{x}|<\mathrm{r}$ выполнено неравенсіго

$$
\left\|\left(1-\chi_{B_{2 r}}(\cdot)\right)(\mathrm{K}(\mathrm{x}-\cdot)-\mathrm{K}(\cdot))\right\|_{\mathrm{M}_{\varphi_{0}}} \leq \mathrm{C},
$$

и интегральный оператор свертки

$$
(K * f)(x)=\int_{R_{\mathbf{a}}} K(x-y) f(y) d y
$$

ограничено действует из $\Lambda_{\varphi}\left(R^{\mathrm{n}}\right)$ в $\mathrm{M}_{\psi}\left(\mathrm{R}^{\mathrm{n}}\right)$, где $\psi(\mathrm{t})=\mathrm{t} \varphi_{0}(\mathrm{t}) / \varphi(\mathrm{t})$, то тогда для любой функции $f \in L_{1}\left(R^{n}\right)$, имеющей компактный носитель, и любого $\mathrm{t}>0$ справедлива оценка

$$
\mathrm{t}(\mathrm{K} * \mathrm{f}) * *(\mathrm{t}) / \varphi_{0}(\mathrm{t}) \leq \mathrm{C}_{1}\|\mathrm{f}\|_{L_{1}},
$$

где постоянная $\mathrm{C}_{1}$ не зависит от $\mathrm{f}(\mathrm{x})$ и $\mathrm{t}$.

() Пелешенко Б.И., 1998 
Доказательство теоремы проводим по схеме доказательства теоремы 2.2 в работе Хермандера [4] с соответствующей модификацией.

Предварительно докажем вспомогательную лемму

Пемма 1. Пусть функции $\varphi_{0}(\mathrm{t}), \varphi(\mathrm{t}), \psi(\mathrm{t}), \mathrm{K}(\mathrm{x})$ и оператор свертки $\left(\mathrm{K}^{*} \mathrm{f}\right)(\mathrm{x})$ удовлетворяют условиям теоремы. Тогда неравенство

$$
\|(\mathrm{K} * \mathrm{f})()\left(1-\chi_{B_{2 r}}(\cdot)\left\|_{M_{\infty}} \leq \mathrm{C}\right\| \mathrm{f} \|_{L_{1}}\right.
$$

выполияется, если

$$
\int_{R^{*}} f(x) d x=0^{\circ} \text { и } f(x)=0 \text { вне куба } B_{r}
$$

с той же постоянной $\mathrm{C}$, что и в (1), для любого куба $\mathrm{B}_{\mathrm{r}}$.

Доказательство. Пусть $r>0$ и функция $f(x)$ из $L_{1}\left(R^{n}\right)$ обращается в нуль вне куба $\mathrm{B}_{\mathrm{r}}$. Так как ядро $\mathrm{K}(\mathrm{x})$ локально-интегрируемо, то свертка (2) существует почти всюду. Используя условие (4), ее можно записать в виде

$$
(K * f)(x)=\int_{R^{n}}[K(x-y)-K(y)] f(y) d y .
$$

Из обобщенного неравенства Минковского для интегралов в симметричных пространствах из [1] и условия (1) получаем неравенство

$$
\|(K * f)(\cdot)\left(1-\chi_{B_{2 r}}(\cdot)\left\|_{M_{* 0}} \leq\right\| K(\cdot-y)-K(\cdot)\left\|_{M_{\infty 0}}\right\| f\left\|_{L_{1}} \leq C\right\| f \|_{L_{1}}\right. \text {. }
$$

Как и при доказательстве леммы 2.1 в работе Хермандера [4] можно убедиться, что полученное неравенство (5) эквивалентно неравенству (1).

Отметим, что из инвариантности неравенства (5) относительно сдвига следует, что для любой функции $\mathrm{f}(\mathrm{x})$ из $\mathrm{L}_{1}\left(\mathrm{R}^{\mathrm{n}}\right)$, имеющей носителем куб $\mathrm{B}_{\mathrm{r}}\left(\mathrm{x}_{0}\right)$ со стороной $\mathrm{r}>0$ и $\mathrm{c}$ центром в точке $\mathrm{x}_{0}$, выполняется неравенство

$$
\|(\mathrm{K} * \mathrm{f})(x)\left(1-\chi_{B_{2 r}}\left(-x_{0}\right)\left\|_{M_{*_{0}}} \leq \mathrm{C}\right\| \mathrm{f} \|_{L_{1}}\right.
$$

c постоянной $\mathrm{C}$, не зависящей оr $\mathrm{f}, \mathrm{r}, \mathrm{x}_{0}$.

Для доказательства теоремы нам понадобится фундаментальная лемма о покрытии, доказанная Кальдероном и Зигмундом. Приведем ее в форме, сформулированной в работе [4].

Лемма 2. Пусть $f(x) \in L_{1}\left(R^{n}\right)$ и число $s>0$. Тогда можно записать

$$
f(x)=v(x)+\sum_{k=1}^{\infty} w_{k}(x)
$$

где $v(x)$ и все $w_{k}(x)$ принадлежат $L_{1}\left(R^{n}\right)$, 


$$
\begin{aligned}
& \|v\|_{L_{1}}+\sum_{k=1}^{\infty}\left\|w_{k}\right\|_{L_{1}} \leq 3\|f\|_{L_{1}} \\
& |v(x)| \leq 2^{n} \cdot s \text { почти всюду }
\end{aligned}
$$

н для некоторых непересекающихся кубов $\mathrm{B}^{(\mathrm{k})}=\mathrm{B}_{\mathrm{r}_{\mathrm{k}}}\left(\mathrm{x}_{\mathrm{k}}\right)$ :

$$
\begin{gathered}
\int w_{k}(x) d x=0, w_{k}(x)=0, \text { если } x \notin B^{(k)}, \\
\sum_{k=1}^{\infty} m\left(B^{(k)}\right) \leq s^{-1} \int_{R^{a}} \mid f(x) d x .
\end{gathered}
$$

Если $f(x)$ имеет компактный носитель, то носители $v(x)$ и всех $\mathrm{w}_{k}(\mathrm{x})$ содержатся в некотором фиксированном компактном множестве.

Доказательство теоремы1. Пусть $f(x) \in L_{1}\left(R^{n}\right)$ имеет компактный носитель. Без ограничения общностиі мы можем считать, что $\|f\|_{L_{1}}=1$. Для упрощения записи положим $\tilde{\mathrm{f}}(\mathrm{x})=(\mathrm{K} * \mathrm{f})(\mathrm{x})$. Функция $\tilde{\mathrm{f}}(\mathrm{x})$ как абсолютно :ходящийся интеграл существует почти всюду и принадлежйт $L_{1}\left(R^{n}\right)$. Разложим $\mathrm{f}(\mathrm{x})$ в соответствии с леммой 2 , тогда неравенство

$$
|\tilde{f}(\mathrm{x})| \leq|\widetilde{v}(\mathrm{x})|+\sum_{\mathrm{k}=1}^{\infty}\left|\widetilde{\mathrm{w}}_{\mathrm{k}}(\mathrm{x})\right|
$$

выполняется почти всюду. Пусть $\overline{\mathrm{B}}$ обозначает куб, полученный растяжением куба В в два раза относительно своего центра. Учитывая условие (9) по лемме 1 (неравенство (6)), имеем

$$
\left\|\widetilde{w}_{k}\left(1-\chi_{k}\left(-x_{k}\right)\right)\right\|_{M_{\varphi_{0}}} \leq C\left\|w_{k}\right\|_{L_{1}}
$$

$$
\operatorname{mes}\left(\bigcup_{k} \bar{B}^{(k)}\right) \leq 2^{n} s^{-1}\|f\|_{L_{1}}=2^{n} s^{-1}
$$

Применяя сначала обобщенное неравенство Минковского [1]; затем неравенства (11) и (7), получаем

$$
\| \sum_{k=1}^{\infty} \widetilde{w}_{k}\left(\chi_{1}-\chi_{\bar{B}_{n_{k}}}\left(-x_{k}\right)\left\|_{M_{* 0}} \leq C \sum_{k=1}^{\infty}\right\| w_{k}\left\|_{L_{1}} \leq 3 C\right\| f \|_{L_{1}}=3 C\right. \text {. }
$$

Так как оператор свертки (3) по условию теоремы ограничен из .$_{\varphi}\left(R_{n}\right)$ в $M_{\psi}\left(R_{n}\right)$, то для функции $\widetilde{v}(x)=\left(K^{*} v\right)(x)$ имеем

$$
\|\tilde{\mathrm{v}}\|_{M_{\psi}}=\mathrm{C}_{0}\left\{\int_{0}^{1 / 2^{\mathrm{n}} s} \mathrm{v}^{*}(\mathrm{t}) \mathrm{d} \varphi(\mathrm{t})+\int_{1 / 2^{\mathrm{n}} \mathrm{s}}^{\infty} \mathrm{v}^{*}(\mathrm{t}) \mathrm{d} \varphi(\mathrm{t})\right\} \text {. }
$$


Из оценки (8) и свойств неотрицательной вогнутой на полуоси $(0, \propto$ функции [1] следует, что правая часть полученного неравенства н: превышает

$$
C_{0}\left\{2^{n} s \varphi\left(1 / 2^{n} s\right)+2^{n} s \varphi\left(1 / 2^{n} s\right)\|f\|_{L_{1}}\right\}
$$

Піусть $\mathrm{t}>0$ и $\tilde{\mathrm{f}}^{* *}(\mathrm{t}) \neq 0, \bar{\varphi}_{0}(\mathrm{t})=\mathrm{t} / \varphi_{0}(\mathrm{t}), \bar{\psi}_{0}(\mathrm{t})=\mathrm{t} / \psi_{0}(\mathrm{t}), \bar{\varphi}_{0}^{-1}(\mathrm{t})$ еств обратная функция к $\bar{\varphi}_{0}(\mathrm{t})=\mathrm{t} / \varphi_{0}(\mathrm{t})$. Выберем $\mathrm{s}^{-1}=2^{\mathrm{n}} \bar{\varphi}_{0}^{-1}\left(8 \mathrm{C}_{0} / \tilde{\mathrm{f}}^{* *}(\mathrm{t})\right)$ тогда с учетом соотношения $t \varphi_{0}(t)=\varphi(t) \psi(t)$

$$
\begin{gathered}
\|\tilde{\mathrm{V}}\|_{M_{\psi}} \leq 2 \mathrm{C}_{0} \varphi\left(1 / 2^{\mathrm{n}} \mathrm{s}\right) 2^{\mathrm{n}} \mathrm{s}=2 \mathrm{C}_{0} \bar{\psi}\left(1 / 2^{\mathrm{n}} \mathrm{s}\right) / \bar{\varphi}_{0}\left(1 / 2^{\mathrm{n}} \mathrm{s}\right) \leq \\
\leq \tilde{\mathrm{f}}^{* *}(\mathrm{t}) \bar{\psi}\left(\bar{\varphi}_{0}^{-1}\left(8 \mathrm{C}_{0} / \tilde{\mathrm{f}}^{* *}(\mathrm{t})\right)\right) / 4
\end{gathered}
$$

Если $\widetilde{\mathrm{f}}^{* *}(\mathrm{t}) / 4 \leq \widetilde{\mathrm{v}}^{* *}(\mathrm{t})$, то отсюда следует неравенство

$$
\tilde{\mathrm{f}}^{* *}(\mathrm{t}) \bar{\varphi}_{0}(\mathrm{t}) \leq 8 \mathrm{C}_{0} \text {. }
$$

Если

$$
\widetilde{\mathrm{f}} * *(\mathrm{t}) / 4>\widetilde{\mathrm{v}}^{* *}(\mathrm{t})
$$

то из неравенства $(10)$ получаем, что $\tilde{\mathrm{f}}^{* *}(\mathrm{t}) \leq \widetilde{\mathrm{w}}^{* *}(\mathrm{t})+\widetilde{\mathrm{v}}^{* *}(\mathrm{t})$ и

$$
3 / 4 \widetilde{\mathrm{f}}^{* *}(\mathrm{t}) \leq\left(\chi_{\mathrm{R}^{\mathrm{a}}-\Omega} \widetilde{\mathrm{w}}\right)^{* *}(\mathrm{t})+\left(\chi_{\mathrm{a}} \widetilde{\mathrm{w}}\right)^{* *}(\mathrm{t})=\widetilde{\mathrm{W}}_{1}^{* *}(\mathrm{t})+\widetilde{\mathrm{W}}_{2}^{* *}(\mathrm{t})
$$

Вначале предположим, что $\tilde{\mathrm{f}}^{* *}(\mathrm{t}) / 4 \leq \widetilde{\mathrm{W}}_{1}^{* *}(\mathrm{t})$. Тогда из неравенства (11) получаем $\widetilde{\mathrm{f}} * *(\mathrm{t}) \bar{\varphi}(\mathrm{t}) \leq 4 \mathrm{C}$.

Затем рассмотрим случай, когда

$$
\widetilde{\mathrm{f}}^{* *}(\mathrm{t}) / 4>\widetilde{\mathrm{W}}_{1}^{* *}(\mathrm{t})
$$

и, следовательно, выполняется неравенство $\tilde{\mathrm{f}}^{* *}(\mathrm{t}) / 2 \leq \widetilde{\mathrm{W}}_{2}^{* *}(\mathrm{t})$. В этом случае из (13) и (14) получаем, что

$$
\widetilde{\mathrm{W}}_{2}^{* *}(\mathrm{t}) \leq \tilde{\mathrm{f}}^{* *}(\mathrm{t})+\widetilde{\mathrm{v}}^{* *}(\mathrm{t})+\widetilde{\mathrm{W}}_{1}(\mathrm{t}) \leq 3 / 2 \tilde{\mathrm{f}}^{* *}(\mathrm{t})
$$

Далее, полагая в неравенстве (12) $\mathrm{s}^{-1}=2^{\mathrm{n}} \bar{\varphi}^{-1}\left(8 \mathrm{C}_{0} / \widetilde{\mathrm{f}}^{* *}(\mathrm{t})\right)$, имеем $\operatorname{mes} \Omega \leq 4^{\mathrm{n}} \bar{\varphi}^{-1}\left(8 \mathrm{C}_{0} / \mathrm{T} \tilde{f}^{* *}(\mathrm{t})\right)$. Из этого неравенства и условия вогнутости функции $\bar{\varphi}_{0}(\mathrm{t})$ следует, что

$$
4^{-\mathrm{n}} \tilde{\mathrm{f}}^{* *}(\mathrm{t}) \bar{\varphi}_{0}(\mathrm{t}) \leq 4^{-\mathrm{n}} \tilde{\mathrm{f}} * *(\mathrm{t}) \bar{\varphi}_{0}(\operatorname{mes} \Omega) \leq \tilde{\mathrm{f}} * *(\mathrm{t}) \bar{\varphi}\left(4^{-\mathrm{n}} \operatorname{mes} \Omega\right) \leq 8 \mathrm{C}_{0}
$$

LIS BCex $0<\mathrm{t} \leq \operatorname{mes} \Omega$. 
Пусть mes $\Omega<t<\infty$. Так как носителем функции $\widetilde{\mathrm{W}}_{2}(\mathrm{x})$ есть множество $\Omega=\bigcup_{k=1}^{\infty} \overline{\mathrm{B}}^{(\mathrm{k})}$, то тогда $\widetilde{\mathrm{W}}_{2}^{*}(\mathrm{t})=0$ для всех $\mathrm{t}>\operatorname{mes} \Omega$. Используя условие вогнутости функции $\bar{\varphi}_{0}(t)$ и неравенства $(15),(16)$, имеем

$$
\begin{aligned}
& \widetilde{\mathrm{f}}^{* *}(\mathrm{t}) \bar{\varphi}_{0}(\mathrm{t}) / 2 \leq \widetilde{\mathrm{W}}_{2}^{* *}(\mathrm{t}) \bar{\varphi}_{0}(\mathrm{t}) \leq \mathrm{t}^{-1} \operatorname{mes} \Omega \widetilde{\mathrm{W}}_{2}^{* *}(\operatorname{mes} \Omega) \bar{\varphi}_{0}\left(\frac{\mathrm{t} \operatorname{mes} \Omega}{\operatorname{mes} \Omega}\right) \leq \\
& \leq \tilde{\mathrm{W}}_{2}^{* *}(\operatorname{mes} \Omega) \bar{\varphi}_{0}(\operatorname{mes} \Omega) \leq 3 / 2 \tilde{\mathrm{f}}^{* *}(\operatorname{mes} \Omega) \bar{\varphi}_{0}(\operatorname{mes} \Omega) \leq 3 \cdot 4^{\mathrm{n}+1} \mathrm{C}_{0} .
\end{aligned}
$$

Таким образом, для любого $\mathbf{t}>0$ выполняется неравенство

$$
\tilde{\mathrm{f}}^{* *}(\mathrm{t}) \tilde{\varphi}_{0}(\mathrm{t}) \leq \max \left\{4 \mathrm{C}, 3 \cdot 4^{\mathrm{n}+1} \mathrm{C}_{0}\right\}
$$

Теорема доказана.

Теорема 2. Пусть функции $\varphi_{0}(\mathrm{t}), \varphi(\mathrm{t}), \psi(\mathrm{t})$ удовлетворяют условию теоремы 1 . Для того чтобы ядро $\mathrm{K}(\mathrm{x}) \in \mathrm{M}_{\varphi_{0}}\left(\mathrm{R}^{\mathrm{n}}\right)$ необходимо и достаточно, чтобы выполнялось условие (1) и оператор свертки ограничено.действовал из $\Lambda_{\varphi}\left(\mathrm{R}^{\mathrm{n}}\right)$ в $\mathrm{M}_{\psi}\left(\mathrm{R}^{\mathrm{n}}\right)$.

Доказательство. Необходимость следует из теорем. 1 и 2 [2]. Достаточность получаем из результатов Крейна - Семенова [1] и неравенства треугольника для нормы пространства $\mathrm{M}_{\varphi_{0}}\left(\mathrm{R}^{\mathrm{n}}\right)$.

Доказанные теоремы дополняют, а в случае $\varphi_{0}(t)=t^{1-1 / a}, \varphi(t)=t^{1 / \%}$, $\psi(\mathrm{t})=\mathrm{t}^{1-1 / \mathrm{q}}, 1<\mathrm{a}, \mathrm{p}, \mathrm{q}<\infty, \mathrm{p}^{-1}-\mathrm{q}^{-1}=1-\mathrm{a}^{-1}$ уточняют теорему 2.2 из работы Хермандера [4] и теорему 3 из работы Степанова [3].

Следующий результат обобщает теорему 2.1 из работы [4].

Следствие. Пуеть выполнены условия теоремы 1. Тогда оператор свертки (2) ограничено действует из симметричного пространства $E\left(\mathrm{R}^{\mathrm{n}}\right)$ в симметричное пространство $\mathrm{F}\left(\mathrm{R}^{\mathrm{n}}\right)$ для всякой пары пространств $\mathrm{E}\left(\mathrm{R}^{\mathrm{n}}\right)$, $F\left(R^{n}\right) c$ фундаментальиыми функциями $\varphi_{E}(t)$ и $\varphi_{F}(t)$, удовлетворяющими условиям $\beta_{\varphi_{0}}<t_{\varphi_{\mathrm{E}}} \leq \beta_{\varphi_{\mathrm{E}}}<\mathrm{l}$.,$\varphi_{\mathrm{F}}(\mathrm{t})=\varphi_{\mathrm{E}}(\mathrm{t}) / \varphi_{0}(\mathrm{t})$.

\section{Библнографические ссылки}

1. Крейн С.Г:, Петунин Ю.И., Семенов Е.М. Интерполяция линейных операторов. М., 1978.

2. ПІелешенко Б.И. О сингуљиных интегральных операторах свертки слабого тита // Тр. MHAH. 1987. T.180. C. $174-175$.

3. Степанов В.Д. Об интеграпьныг операторах свертки // Докл. АН СССР. 1978. Т. 243, №1. C. $\quad$.5-48.

4. Хермандер Л. Оцевки для операторов, инвариантных относительно сдвита. М., 1962. 